\title{
GEM : Requirement-driven Generation of ETL and Multidimensional Conceptual Designs
}

\author{
Oscar Romero \\ Universitat Politècnica de \\ Catalunya, BarcelonaTech \\ Barcelona, Spain \\ oromero@lsi.upc.edu
}

\author{
Alkis Simitsis \\ HP Labs, Palo Alto, CA, USA \\ alkis@hp.com
}

\author{
Alberto Abelló \\ Universitat Politècnica de \\ Catalunya, BarcelonaTech \\ Barcelona, Spain \\ aabello@essi.upc.edu
}

\begin{abstract}
At the early stages of a data warehouse design project, the main objective is to collect the business requirements and needs, and translate them into an appropriate conceptual, multidimensional design. Typically, this task is performed manually, through a series of interviews involving two different parties: the business analysts and technical designers. Producing an appropriate conceptual design is an errorprone task that undergoes several rounds of reconciliation and redesigning, until the business needs are satisfied. It is of great importance for the business of an enterprise to facilitate and automate such a process. The goal of our research is to provide designers with a semi-automatic means for producing conceptual multidimensional designs and also, conceptual representation of the extract-transform-load (ETL) processes that orchestrate the data flow from the operational sources to the data warehouse constructs. In particular, we describe a method that combines information about the data sources along with the business requirements, for validating and completing -if necessary- these requirements, producing a multidimensional design, and identifying the ETL operations needed. We present our method in terms of the TPC-DS benchmark and show its applicability and usefulness.
\end{abstract}

Keywords: DW, ETL, Multidimensional Design, Conceptual Design.

\section{INTRODUCTION}

"A gemstone or gem is a piece of attractive mineral, which -when cut and polished- is used to make jewelry or other adornments. Most gems are hard, but some soft minerals are used in jewelry because of their lustre or other physical properties that have aesthetic value." (Wikipedia)

As most of the raw materials and resources, gems are out there in large varieties and quantities, but we need to dig and work hard in order to get them and make profit out of them.
Data are the gems of the enterprise. They are available at large quantities, but we need to "dig" for recognizing the relevant and useful ones, and to adjust and polish them for making our valued assets, our "jewelry". The jewelry for an enterprise is any tool or means that facilitates strategic decision making and helps in satisfying business needs. Such a tool is a data warehouse (DW) that organizes the raw, source data in a way that enables decision support. Building a DW requires two essential constructs: the multidimensional (MD) design of the target data stores and the extracttransform-load (ETL) process that populates the target data stores from the source ones.

Nowadays, the construction of conceptual MD and ETL designs is an error-prone, manual process that undergoes several rounds of reconciliation and redesigning, until the business needs are satisfied. It is essential for the business of an enterprise to facilitate, speed up, and automate these designs process.

This paper presents a system called GEM (Generating $E$ tl and $M$ ultidimensional designs). GEM starts with a set of source data stores and business requirements -e.g., business queries, service level agreements (SLAs) - and based on these, it produces a MD design for the target data stores, along with a set of ETL operations required for the population of the target DW.

The semantics, characteristics, and constraints of data sources are represented by means of an OWL ontology. The business requirements are expressed in a structured form. We consider functional requirements that drive the generation of the MD design constructs and also, soft or nonfunctional requirements - e.g., freshness, recoverability, availability- that can be used for giving "lustre" and adding value to our designs. For example, based on a freshness requirement we may decide which data source to use and according to a recoverability requirement we may choose to enrich the ETL process with recovering techniques.

For each business requirement, we identify the relevant part of the data sources (e.g., concepts, attributes, properties). If we identify conflicts, we either suggest corrections or ask for user feedback. The output of these tasks is an annotated subset of the source ontology that corresponds to a business requirement. Next, we classify the relevant concepts as dimensional or factual and validate the result. We also explore schema information for identifying the respective ETL operations. Finally, we consolidate the individual designs, one for each business requirement, and get the conceptual MD and ETL designs. 
Contributions. In particular, our main contributions are as follows.

- We present GEM, a system that facilitates the production of ETL and MD designs, starting from a set of business requirements and source data stores. To the best of our knowledge, GEM is the first approach towards the semi-automatic generation of both the ETL and MD conceptual designs.

- We propose algorithms for computing closure, finding and validating an ontology subset as a MD schema, and identifying ETL operators.

- We evaluate our method using the schema and constructs of the TPC-DS benchmark and show the quality of the GEM designs.

Outline. The rest of the paper is structured as follows. Section 2 formulates the problem at hand and presents the GEM architecture. Sections 3 and 4 discuss the validation and completion of business requirements, respectively. Then, Section 5 describes the validation of the MD design and Section 6 the identification of ETL operations. Section 7 presents the conciliation of designs corresponding to individual business requirement. Section 8 evaluates GEM using the TPC-DS benchmark and Section 9 presents the related work.

\section{GEM IN A NUTSHELL}

This section gives an overview of our system, GEM. Given two inputs, namely information about the operational sources and a set of user requirements, our system produces two designs: one that represents the MD design of the target DW constructs and one that represents the conceptual ETL flow that interconnects the target constructs to the operational sources.

\subsection{Inputs}

Source data stores. We capture the semantics of the data sources in terms of an OWL ontology. In previous work, we have shown that a variety of structured and unstructured data stores can be elegantly represented as graphs, and we have described how we can construct an appropriate ontology for such data stores by integrating a domain vocabulary with the data sources' vocabulary [18]. Here, due to space consideration, we assume that we do have an OWL ontology annotated with the semantics of the operational data stores for each concept and property. For further details on how we get this ontology from the sources, we refer the interested reader to our past work [18]. Figure 3 (page 8) depicts an example ontology based on the TPC-DS schema [20].

Business requirements. In typical DW and ETL engagements, the design starts from a set of functional and non-functional requirements (respectively f-req and nf-req, from here on) expressing business needs. Example requirements could be "examine stocks provided by suppliers" or " $a$ report on total revenue per branch should be updated every 10 minutes". Such requirements often come as service level agreements (SLAs) or business queries and are expressed in various forms, either structured or unstructured. Much work has been done in capturing and representing business needs. For example, SLAs expressed as free-form text, require natural language processing (NLP) techniques for being interpreted in a machine processable way. How to capture such requirements are out of the scope of this work. Here, without loss of generality, we consider requirements expressed in a structured way (e.g., by means of $i^{*}$ profiles [22]). Such requirements can be represented in an XML file of a specific structure.

This XML file contains two main parts. The first part involves functional or information requirements that are captured by identifying the measures and dimensions of interest. For example, stocks would be the measure and suppliers the dimensional concept.

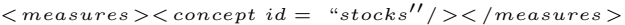

The second part, involves the non-functional requirements of interest for each concept indicated by the functional requirements. For example, the measures used by the revenue report (i.e., the respective view) should conform to a nonfunctional requirement for freshness that requires that the corresponding data should be updated at least every $10 \mathrm{~min}$ utes.

$$
\begin{aligned}
& \left.<\text { concept id }=\text { "v_revenue" }><n f_{-} r e q\right\rangle \\
& <\text { freshness format }=" H \text { H 24:MI:SS" > \&lt;00:10:00 </freshness }>
\end{aligned}
$$

Although a detailed description of the XML structure for representing such requirements is prohibited due to space consideration, its content is as follows.

- Levels of detail, which represent data granularity. The user may provide a discretization process for continuous (or with high cardinality) data types.

- Descriptors, which carry out selections over them (i.e., slicers). Type of comparison carried out; e.g., "year = YYYY".

- Measures, which should be analyzed. Aggregation function and a partial order between them; the latter is needed when we perform different aggregations (one order per dimension). In doing so, we would be able to distinguish between, for example, 'average of sums' and 'sum of averages'.

We may also have non-functional requirements for each one of the above three or even for the whole design. All in all, the expressivity we may capture is equivalent to that of the dimensional expressions introduced in [5], although our XML structure is conceived to capture multidimensional requirements over a domain (i.e., non-multidimensional) ontology.

As a remark, different requirements affect different design levels. For example, a freshness requirement indicates how often an ETL flow should run in order to meet the required latency in updating the DW. However, such decision affects the execution level and should be taken under consideration at the physical model. Nevertheless, we may need to use this requirement during the conceptual design as well. For example, assume two source data stores containing the same data but placed in different locations for business reasons (e.g., two snapshots placed in two different branches of the organization). Assume also that the first data store is updated every hour and the second every 5 minutes or that the congestion of the network coming from the first data store is significantly greater than the one coming from the second source. If we have such information, then based on the freshness requirement we need to honor for our target data stores, we should decide to pull data from the second data 


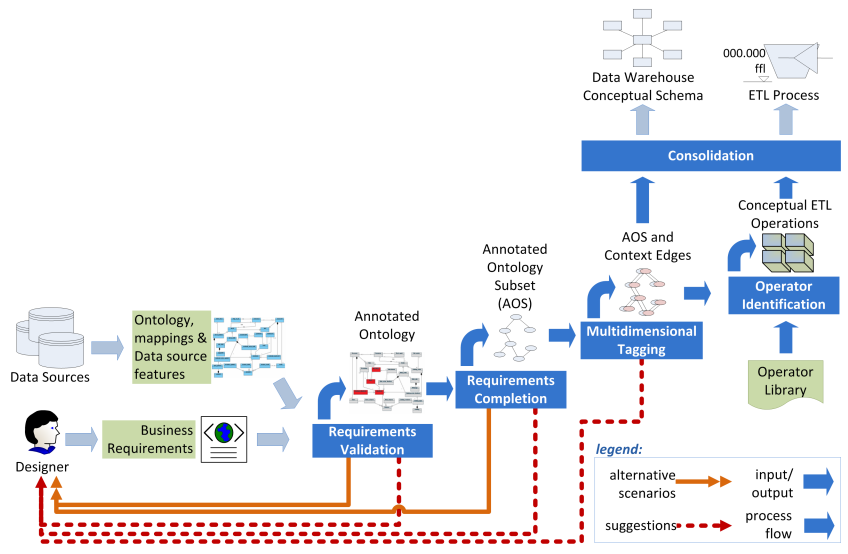

Figure 1: System architecture

store. Clearly, such decision is to be taken at the conceptual level.

However, we are interested in capturing all requirements. Those that cannot be used at the conceptual level (which is the focus of this paper) should be transferred to the subsequent, more detailed design levels, along with the outcome of this process; i.e., the conceptual ETL and MD designs. Hence, the designer of the logical and physical models does not need to revisit and reinterpret the original set of business requirements.

\subsection{System Architecture}

The process of producing the ETL and MD designs is a semi-automatic process comprising five main stages (see Figure 1).

Stage 1: Requirement validation. First, the system checks if there is a mismatch among the business requirements (either functional or non-functional) and the data sources. In case of mismatch, it identifies the possible problems or it may suggest relaxation of the requirements.

Stage 2: Requirement completion. After considering the business requirements, the system complements them with additional requirements based on the information gathered from the sources. This stage identifies intermediate concepts that are not explicitly stated in the business requirements, but are needed in order to answer the f-reqs. Hence, the system may suggest new analysis dimensions or facts of interest to the user. User feedback is welcomed for ensuring correctness and compliance to the end-user needs.

Stage 3: Multidimensional tagging. Next, we tag the concepts identified by the previous stages, as either factual or dimensional and validate the correctness of the f-req tagging according to MD design principles. Hence, we check two issues: i) first, whether the factual data is arranged in a MD space (i.e., if each instance of factual data is identified by a point in each of its analysis dimensions) and second, ii) whether the data summarization is correct by examining whether the following conditions hold [9]: (1) disjointness (the sets of objects to be aggregated must be disjoint); (2) completeness (the union of subsets must constitute the entire set); and (3) compatibility of the dimension, the type of measure being aggregated and the aggregation function.

Stage 4: Operator identification. The ETL operations are identified in three phases. First, we use the annotations generated by the previous steps for extracting schema modification operations. Then, we complement the design with additional information that might be found in the sources and with typical ETL operations regarding surrogate key and slowly changing dimensions.

Stage 5: Conciliation. The previous stages run once for each f-req. Eventually, the individual results obtained per f-req are conciliated in a single conceptual MD schema and a single ETL flow.

\subsection{Output}

At the end, we produce a conceptual, MD schema composed by facts and dimensions. In addition, we identify the ETL operations needed in order to interconnect the source data stores to the MD constructs.

\section{REQUIREMENT VALIDATION}

Starting from the inputs discussed in Section 2.1, we validate the business requirements w.r.t. the available data sources, as follows: (a) we tag the ontology concepts corresponding to the f-req at hand and identify any mapping conflicts, and (b) we include and then validate assertions regarding nf-reqs and the data sources features.

Validating f-req. The input XML file contains three kinds of concepts: measures, levels, and descriptors (see Section 2.1). So, first, we tag the concepts in the input ontology with corresponding labels. Then, we check whether the tagged concepts can be mapped to the sources (either directly or by means of ETL operators). When an error occurs, we ask for user feedback. Due to space consideration we omit the formal description of the algorithm. A sketch of the algorithm is as follows:

(1) If a tagged concept is mapped to the sources (i.e., it has an annotated mapping) then no further action is needed.

(2) Else, if the tagged concept is involved in a concept taxonomy: (2.1) if any of its subclasses have a mapping, we annotate the tagged concept with 'renaming' and 'union'. (2.2) Otherwise, if any superclass has a mapping, we use the general concept mapped and tag the required concept with 'renaming' and 'selection' (if a discriminant function has not been specified in the input XML file then, user feedback is required). If the tagged node has several superclasses, then 'minus' or 'intersection' are also considered. We detail these in Section 6.

(3) Else, if exists a (transitive) one-to-one association to a mapped concept then, GEM suggests it as a potential synonym. If the suggestion is accepted, the f-req is updated with the synonym concept.

(4) In any other case, the concept is not available in the data sources.

Validating nf-req. Nf-req are used either for helping with making design choices during the conceptual design or for annotating respective design constructs (e.g., operators, concepts, even flows), so that they can be used in later, more detailed designs (i.e., the logical and physical models). As discussed earlier, an nf-req is attached to a specific concept or property (if it concerns a subflow or the whole ETL flow, then it involves a part of the annotated ontology and it will be used in later designs). Thus, we treat them in a similar way as the functional requirements.

Recall the example from Section 2.1: "freshness $<10 \mathrm{~min}$ for the revenue report". Assume that this report maps to a source concept sales (based on the view definition that populates this report) and also that sales has a property update_freq with value "every $30 \mathrm{~min}$ ". Then as we map the 
nf-req to the source, we check whether freshness is satisfied with the update_freq (it does not in this case) and we proceed accordingly.

For doing such mappings, we need to know how the nf-req relate to source properties or features. For example, here, we should know the relationship <freshness, update_freq $>$. Similarly, we may have $<$ freshness, $f$ (network_congestion) $>$ (that is a function over network_congestion), $<$ freshness,estimated_extraction_time $>$, and so on. For handling such issues, we assume a vocabulary that relates nf-req to quantitative metrics. For a more detailed discussion on mapping qualitative to quantitative metrics, we refer the interested reader to [3].

If we find a problem (like in this example), we inform the designer. By reasoning, we are also able to identify alternative scenarios involving relevant sources. For example, a mirror source concept about sales residing on a different location.

\section{REQUIREMENT COMPLETION}

This stage takes as input the annotated ontology produced in the previous stage and it completes the requirements regarding the sources. First, it identifies intermediate concepts that are not explicitly stated in the f-req, but needed to retrieve the required information. If an f-req cannot be met, it suggests alternative solutions. Finally, it produces the ontology subset needed to answer the business query at hand and additional annotations regarding ETL operations.

This stage starts with a pruning process. We identify how tagged concepts are related in the sources and then, (a) we disregard concepts/relationships not mapped nor tagged (if a concept taxonomy is affected, we replace the concept pruned with the first superclass mapped/tagged); and next, (b) we prune all the mapped many-to-many (i.e., *_*) associations. Note that such associations violate the three summarization necessary conditions [9] and thus, they cannot be exploited for MD design. The outcome of this pruning is a subset of the input annotated ontology, which we call AOS. Since an arbitrary ontology can be represented as a graph, we will talk about paths between concepts and thus, we will also refer to concepts as nodes and to associations as edges.

Looking for Paths Between Tagged Concepts. For identifying how tagged concepts are related in the sources, we use the following algorithm that computes paths among tagged concepts.

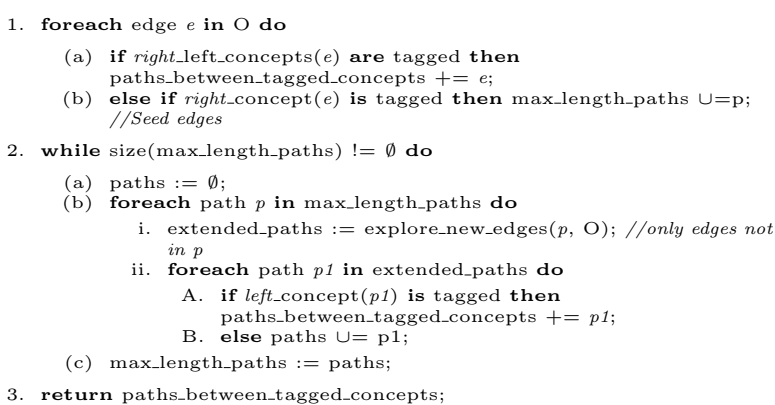

We start by identifying edges directly relating tagged concepts (step 1a) and edges reaching tagged concepts (from now on, seed edges; step 1b). For the sake of understandability, although the AOS has no directed edges, we say that the tagged node is in the edge right-end, and its counterpart to be in the the left-end. The rest of the algorithm is an application of the transitive property but starting from tagged concepts. At the first iteration, we explore new edges such that their right-end matches the left-end of a seed edge, and similarly for the forthcoming iterations (step 2(b)i). Intuitively, we explore paths starting from tagged concepts by exploring a new edge per iteration. This guided exploration has two main restrictions: we cannot explore any edge already explored in a given path (step 2(b)i) and if we reach another tagged concept we finish exploring that path (i.e., we have found a path between tagged concepts; step 2(b)iiA). Note that in a given iteration $i$, we only explore the longest paths computed in the previous iteration (steps $1 \mathrm{~b}$ and $2 \mathrm{c}$ ). Eventually, we explore all the paths and the algorithm finishes (step 2).

Observe that step 1 can be computed by means of generic reasoning. The transitive closure, in general, cannot be computed by using generic reasoning, but the specific closure for certain (tagged) concepts can be simulated using generic reasoning (similar to the idea introduced in [16]).

This algorithm is sound since it computes direct relationships and propagates them according to the transitivity rule and complete, because it converges (note that each edge is explored only once). This algorithm has a theoretical exponential upper bound regarding the size of the longest path between tagged concepts. However, this theoretical upper bound is hardly achievable in real-world ontologies as they have neither all classes with maximum connectivity nor all paths are of maximum length. Moreover, note that *_* relationships were previously pruned (see our evaluation in Section 8 too).

Producing the Output Subset. Based on the paths between tagged concepts that the previous algorithm found, next algorithm determines the ontology subset needed to answer the f-req.

(1) If between two tagged concepts there are more than one path, we ask the user for disambiguation: i.e., which is the path fulfilling the semantics needed for the f-req at hand (note that the user may not select any).

(2) For each pair of related tagged concepts not involving a descriptor, edges forming that path are annotated as aggregation edges, because these relationships determine the data granularity of the output.

(3) The annotated ontology subset is compound by the paths selected in (1). Note that these paths include the intermediate concepts (i.e., those not tagged but involved in the paths). At this point, taxonomies are also disregarded.

Annotating the Ontology Subset. Having an AOS containing the concepts needed for the f-req, we check whether the relationships captured make MD sense regarding the tags.

We check the semantics of each edge (according to the tag -if any- of the concepts it relates and its multiplicity) and according to these semantics, we label each edge with those MD relationships it could represent (i.e., the MD concepts it could relate). From here on, we consider factual nodes (those tagged as measures) and dimensional nodes (those either tagged as levels or descriptors). In order to guarantee the MD design principles (see Section 2.2) factual and dimensional nodes must be related properly. For example, factual data cannot be related to dimensional data by means of a one-to-many (i.e., 1-*) association, as by definition, each instance of factual data is identified by a point in each of its analysis dimensions. In short, dimensional data can only 


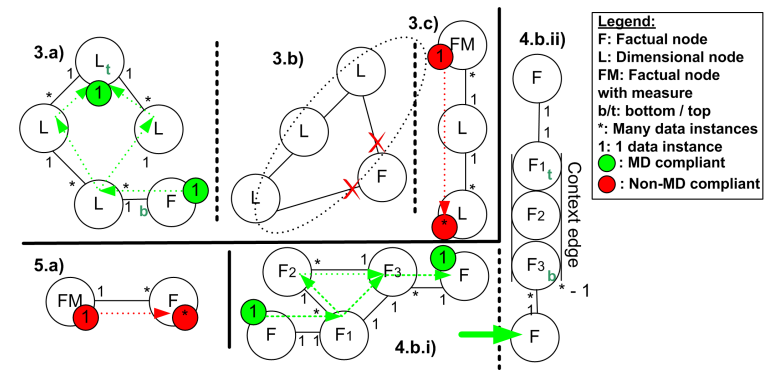

Figure 2: Graphical representation of the multidimensional validation steps

appear in the ${ }^{*}$-end of an edge when the other end is also tagged as dimensional data. Furthermore, non-complete associations (i.e., accepting zeros) in the dimensional end are not allowed either, as they do not preserve completeness. A detailed discussion on edge semantics can be found in [15].

Thus, we analyze the graph looking for edges not making MD sense and try to fix them. For example, if the node in the *-end of a ${ }^{*}-1$ association is tagged as dimensional then, its counterpart must also be dimensional. If by doing so we have been able to infer an unequivocal label, this knowledge is propagated in cascade to the rest of the AOS. However, if we identify a meaningless conceptual relationship (i.e., both ends are tagged in a forbidden way), the algorithm stops and alternative analysis scenarios are proposed according to [14].

\section{MULTIDIMENSIONAL VALIDATION}

This stage validates the AOS and checks whether its concepts and associations collectively produce a data cube. If the validation fails (according to the constraints discussed in Section 2.2), we propose alternative analysis solutions. Otherwise, the resulting MD schema is directly derived from the AOS.

The previous stage might have propagated some tags when labeling the AOS associations (i.e., inferring unequivocal knowledge), but it does not guarantee that all the concepts have an MD tag at this point. Thus, we start this stage with a pre-process aimed at deriving new MD knowledge from unlabeled concepts, and each unlabeled concept is considered to play a dimensional role or a factual role. Furthermore, it would be possible to relabel a dimensional node as dimensional/factual node. Next, we validate if any of these labels, eventually, make MD sense. Thus, this step determines every potential MD labeling making sense for the input f-req and we determine how these alternatives would affect the output schema, deriving (in some cases) interesting analytical options that may have been overlooked by the user.

For each possible combination of new labels, an alternative annotation is created if the labels do not contradict the edge semantics already depicted in the AOS. Subsequently, each of these AOS will be validated and only those that make MD sense will be finally considered. Therefore, an f-req could produce several valid MD labelings for the same AOS and thus, multiple MD schemas.

The validation process introduced in this stage guarantees the multidimensional normal forms presented in $[7,8]$ for validating the output MD schema, and the summarizability constraints discussed in [11]. This algorithm is called once for each alternative labeling generated.

1. If ! factualdata $(A O S)$ then

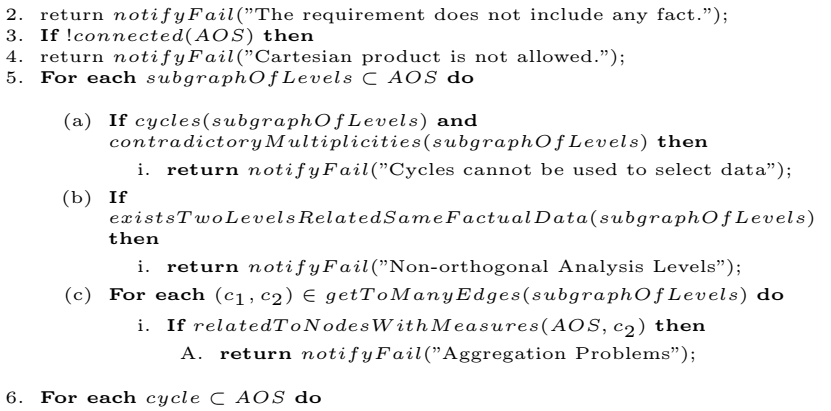

6. For each cycle $\subset$ AOS do

(a) If contradictoryMultiplicities(cycle) then

(b) else se

i. askUserForSemanticValidation();

add(AOS, newContextEdge(bottom(cycle), top(cycle), cycle));

7. For each $\left(c_{1}, c_{2}\right) \in \operatorname{getToManyEdges}(A O S)$ do

(a) If relatedToNodesWithMeasures $\left(A O S, c_{2}\right)$ then i. return notifyFail ("Aggregation problems between
Measures");

Step 1 ensures that the AOS contains factual data. Note that in our pre-process we could have labeled nodes as factual data that do not contain measures (from here on, we distinguish between factual nodes and factual nodes with measures). So this function returns false if all the nodes are labeled as dimensional data. Step 3 ensures that the AOS is connected to avoid "Cartesian Product".

The intuition behind steps 5 to 7 is shown in Figure 2 . Step 5 validates levels subgraphs (i.e., subgraphs only containing level concepts) with regard to where factual nodes are placed. Firstly, every subgraph must represent a valid dimension hierarchy (we must be able to identify two nodes in the level subgraph which represent the top and bottom levels of the hierarchy (Step 5a)). Two different levels in a subgraph cannot be related to the same factual node (Step 5b). Moreover, level - level edges raising aggregation problems in factual nodes with measures must be forbidden (Step $5 \mathrm{c}$, where by convention we assumed that in every $*-1$ edge $\left(c_{1}, c_{2}\right), c_{1}$ corresponds to the ${ }^{*}$-end of the association). Thus, this step validates the correspondences between dimensional nodes, whereas Step 6 validates the path of factual nodes (MD data retrieved) as a whole (i.e., validates cycles in the path of factual nodes to ensure that they are not used to select data, similarly to the validation of levels cycles in 5a). Once the cycle has been validated, the edges involved are clustered in a context edge (from bottom to top of the cycle, since cycles are checked to correspond a correct multi-path aggregation hierarchy, i.e., a one-to-many lattice) labeled with the cycle multiplicity, as shown in Figure 2. Finally, step 7 looks for potential aggregation problems induced by factual nodes with measures at the 1-end of a 1-* edge (either context edge or not).

This stage will eventually have validated each graph as a data cube.

\section{OPERATION IDENTIFICATION}

Operation identification is a semi-automatic process that comprises three phases.

Phase I. This phase identifies operations that are needed for mapping the source to target data stores, using the target schema produced in the previous stage. For example, for aggregating over states, we need a location dimension at the 
target site and to map it with source information about zip code, street address, and so on.

During this phase, we identify mainly schema modification operations as follows. Selection is generated from concepts having attached a selection condition: from slicers recorded in AOS; or when a required concept does not have any mapped source (neither it nor its subclasses), while some of its superclasses do have such mapping. Union appears when a required concept is not directly mapped to the sources, but some of its subclasses are. Similarly, Intersection and Minus are generated when a concept is not mapped but some of its superclasses are. Join is generated for every association in the ontology; if one or both of the association ends is not mandatory, we state it as outer. Aggregation is generated when a $*_{-} 1$ association is found so that there is a measure at its *-end. Renaming is generated for each attribute in the data sources and gives to it the name of the corresponding ontological concept. Projection is generated for each concept and association in the ontology. Function expresses operations stated in the requirements, like a discrimination process for an attribute to be used in a dimension or a transformation for an attribute to facilitate its interpretation as a measure.

Starting from the AOS, we iteratively synthesize several of its nodes into one single operation, as follows.

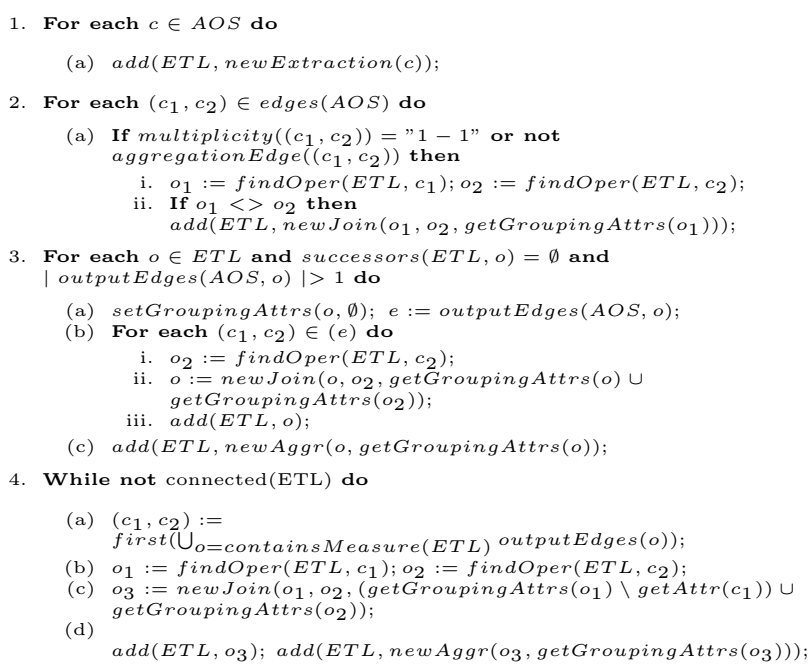

The ETL variable is a directed acyclic graph that tracks the ETL flow generated, whereas the findOper (ETL $g$, concept $c$ ) function looks for a node in $g$, with no successors, such that it contains $c$. Step 1 considers extraction operations like a single table access, a union, an intersection or a minus operation, along with the corresponding selection, projection, renaming mechanisms, and functions. Step 2 fuses all data that do not involve any aggregation. Hence, for those AOS nodes related by means of 1-1 associations (i.e., identity), we join their corresponding operations in the ETL. We also join nodes connected with edges that do not involve aggregation (i.e., stemming from slicing requirements and identified in Section 4).

Step 3 creates the basic cubes. First, we check the already generated operations that have no successors, and whose AOS nodes have more than one edge with the 1-end related to a concept in another ETL node without successors (observe that after step 2 only ${ }^{*}-1$ associations remain). Next, we successively join these operations. The grouping attributes of the final operation is the union of the grouping attributes of each joined operation. Note that a grouping operation is generated to guarantee that data is at the appropriate granularity.

Finally, step 4 connects all cubes produced, starting from those with measures, by following the order specified by the requirements. Since each AOS edge not used yet corresponds to an aggregation, we join the output of the operations (following the AOS aggregation edges), substitute the grouping attributes of $c_{1}$ by those of the new aggregation level $c_{2}$, and generate the grouping operation taking into account the new attributes. The choice of the aggregation function depends on the requirements (there, it should be associated to a corresponding measure and $c_{2}$ ) or a default one is used; e.g., $S U M$.

Phase II. During this phase, the designer might want to refine the design produced by checking for additional information at the sources that might be useful. (Part of this phase can be done before Phase I too.) For example, the domain ontology might relate state with zip code and street address. If there is a source containing information about "location" and contains both the street address and zip code in the same field, then such information is definitely useful, but the domain ontology cannot help. We can correct this by enriching the result with such a mapping and producing the appropriate function(s).

$\mathrm{Nf}$ reqs can be exploited in a similar way. For example, a strict requirement regarding recoverability may suggest to consider adding recovery points at points of the flow that are generally known for being expensive (e.g., after the extraction phase or after an expensive blocking operator [17]). Of course the final decision on which are the good places to add recovery points is to be taken by an optimizer at the logical level [17].

The same holds when we work with f-reqs that involve the data itself. For example, a requirement like "make sure that each customer is considered once" can add a "de-duplicate customer info" operation to the design.

Phase III. The last phase complements the design with operations needed to satisfy standard business and design needs. This task is mainly automatic and involves typical DW operations that can be identified and added to the design after the consolidation phase.

For example, common practices suggest replacing production keys with surrogate keys. For that, the system identifies the respective production keys and enriches the design with appropriate 'surrogate key assignment' operations. Similarly, the system adds operations that take care of slowly changing dimensions (SCDs). There are standard dimensions that are not updated very often (e.g., dimensions that keep structural information about the organization such as geographical location, customer information or product information). Hence, the design can be enriched with operations that handle the update of such dimensions. Possible update operations for SCDs can be: do nothing (do not propagate changes), keep no history (overwrite old values with new data), keep history by creating multiple records in the dimensional tables with separate keys, keep history using separate columns, keep history by storing new data to an active table and keep (all or some of the) old values to 'history tables', or use a hybrid approach. Of course, here we list just a few frequently used operations. The list can go long and our method is extensible to adapt such a list. 


\section{CONCILIATION}

Results obtained for each input f-req are conciliated to produce (a) a minimal constellation schema subsuming each of the conceptual schemas obtained, and (b) an ETL flow subsuming all the ETL processes. Next, we sketch the respective algorithms; their formal descriptions are omitted due to space constraints.

Conciliation for MD. Graphs produced for each f-req (i.e., conceptual schemas obtained) are processed as follows. First, GEM looks for all the factual nodes identified in the input graphs, and creates a new factual class for each one (every class will eventually produce a MD schema at the end of the conciliation process). Measures are added as attributes to the corresponding class.

Next, we conciliate the dimension hierarchies identified for each f-req. We first conciliate compatible hierarchies (two hierarchies are compatible if they share their atomic level). For these, we check the hierarchies graphs that contain the nodes forming the dimensions as follows: (a) Let $h$ and $h$ ' be two hierarchy graphs. If $h$ is equivalent to $h$ ' (except for their descriptors), we merge both hierarchies. (b) If $h$ subsumes $h$ ' and $h$ ' does not subsume $h$, the descriptors of $h^{\prime}$ are mapped to $h$, and $h^{\prime}$ is removed from the set. (c) If $h$ does not subsume $h^{\prime}$ and $h^{\prime}$ does not subsume $h$, they are conciliated as follows: we conciliate the overlapping part they share (by keeping the common structure and aligning their descriptors) and we consider two alternative branches in the resulting hierarchy, one branch for each disjoint part of the subgraphs.

The conciliated dimension hierarchies and those that are not compatible with any other are depicted in the MD schema. At the end, we obtain a star-schema for each factual class identified. Note that conciliated dimensions enrich the conceptual schema; they provide other factual classes with new analytical perspectives considered in other f-reqs. Clearly, this process does not introduce any summarizability problem, because we only merge compatible labels.

Conciliation for ETL. We work similarly for merging ETL flows. Each ETL flow is a DAG and we want to create a large graph (possibly disconnected) containing all the individual ETL flows. Starting from a target table, we build the flow that connects it with the sources. We iterate to resolve the flows for all targets. However, the flows might contain overlapping parts. Identifying these is an optimization challenge, which can be seen as a graph matching problem [2].

\section{EVALUATION}

We evaluated GEM using the TPC-DS benchmark [20]. TPC-DS provides a set of DW tables - both facts and dimensionsalong with a set of data sources. ETL operations (called data maintenance functions) are also provided, for maintaining fact tables and dimensions. Finally, a set of business queries (i.e., business requirements) exists. Having all these constructs allows us to evaluate our method as follows. Starting only from the business queries and the data source, we use GEM for producing the DW schema and ETL operations. Then, we compare our solutions to the design constructs provided by the benchmark. Here, due to limited space, we show results concerning the store_sales cube (the results generalize throughout the whole benchmark though).

We worked as follows. We constructed an ontology containing all source tables, specializations, and we added a few additional concepts that do not map to the data sources. Thus, we intentionally make the ontology more complex by adding more classes to stress GEM; note, that adding more associations does not affect our system, since these will be pruned during the creation of AOS.

First, we examine the search space produced for the AOS creation. Figure 4 presents the number of algorithm iterations needed to converge, the total number of paths computed, the number of paths between tagged concepts (i.e., the output) and the maximum length of the output, per business query. It shows that the search space is not exponential regarding the length of the longest path. Indeed, although the average length of the longest path is 8 , our algorithm computes no more than 178 paths (24 between tagged concepts) in the worst case. These findings verify the feasibility and efficiency of our approach in real-world cases. In fact, the worst total time did not exceed $900 \mathrm{~ms}$. Constructing AOS is the most expensive part of our method; the rest tasks are processed fairly fast, in much less time.

Next, we evaluated the quality of our solutions (see Figure 5). Every business query reveals a part of the final design (tables and attributes). Frequently, business queries reveal overlapping information. However, after a few iterations over these queries (in fact, after the fifth query) we identified correctly all target tables. Since numerous attributes are involved overall, identifying them requires digging into more requirements. After processing 11 business queries, we identified almost $40 \%$ of the total attributes. However, attributes are added throughout the whole process. For example, surrogate keys are identified after Phase III of the ETL operation identification task.

Two observations can be made at this point. One may find tempting the fact that the target tables are identified really fast. Thus, after a certain point of her choice, the designer might want to stop this automatic process and start refining the design by herself. As an aside issue, many business queries involve the same target design constructs. This means that these constructs (e.g., tables) should be quite popular and this information can help us in the physical design; e.g., for choosing indices or partitioning schemes.

Similar are the findings for the identification of ETL operations (see Figure 6). GEM returned almost $60 \%$ of ETL operations after the completion of Phase I. The remaining operations (not shown in the figure) are mostly surrogate key assignments and a few SCDs, which are identified after Phase III. Therefore, GEM identifies the complete set of ETL operations for the TPC-DS case.

\section{RELATED WORK}

Various efforts have been proposed for the conceptual ETL modeling. These include approaches based on ad hoc formalisms [21], on standard languages like UML (e.g., [10]), MDA (e.g., [12, 13]), BPMN [1], and on semantic Web technology and graph transformations [18]. None of these efforts considers synchronous creation of MD design.

Many works have dealt with designing DW models; e.g., $[4,6,12,14,19]$, to mention a few, but the list is long. However, in most works, it seems that the more the process gets automated, the more the integration of requirements is overlooked on the way. Recently, the use of ontologies was considered for facilitating this task [14]. However, that work aims at identifying the MD knowledge contained in the sources and overlooks business requirements. Another 


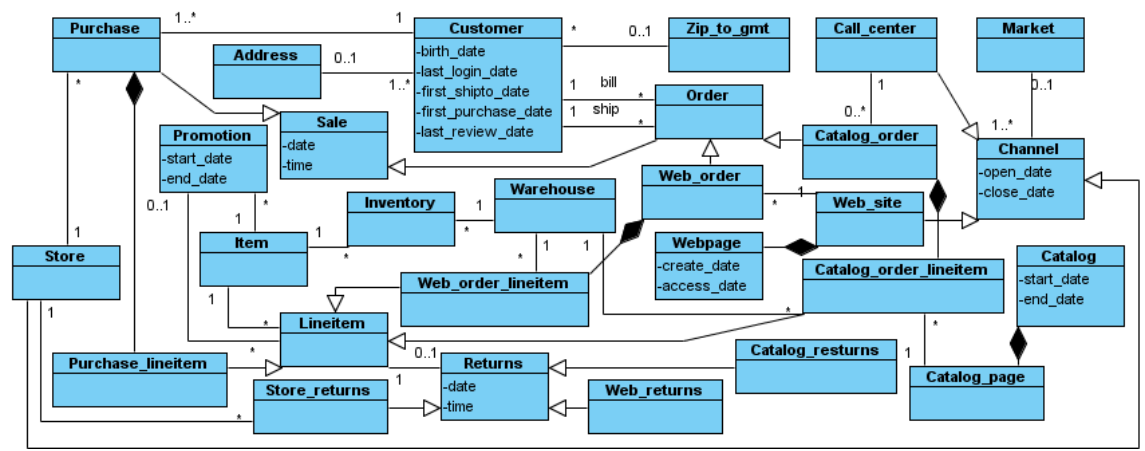

Figure 3: Ontology for TPC-DS data sources

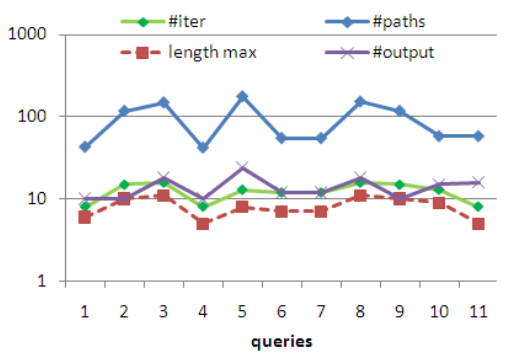

Figure 4: Space

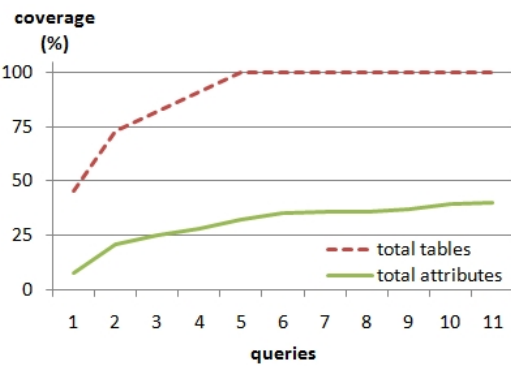

Figure 5: MD coverage

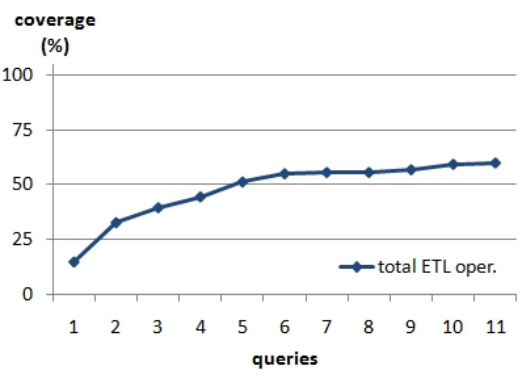

Figure 6: ETL coverage approach to MD design considers business requirements too [15], but the f-req are considered in the form of SQL queries, so a major design task is done manually. GEM automates this part and automatically creates such queries from f-req. In addition, GEM is different from all previous approaches in that it identifies the ETL operation at the same time.

\section{CONCLUSIONS}

We have presented GEM. A system that facilitates the (semi-)automatic generation of ETL and MD conceptual designs, starting from a set of business requirements and data sources. In particular, we have described how the requirements can be validated and enriched, in order to produce an annotated ontology containing correct information for both the sources and the requirements. Then, we have shown how to use this ontology for producing the MD and ETL conceptual designs. Finally, we have reported on our experimental findings working on the TPC-DS benchmark. Our future plans involve extending our techniques to the logical and physical levels.

\section{1. z. REFERENCES} using BPMN and BPEL. In DOLAP, pages 41-48, 2009

[2] T. H. Cormen, C. E. Leiserson, R. L. Rivest, and C. Stein. Introduction to Algorithms. The MIT Press, 2001.

[3] U. Dayal, M. Castellanos, A. Simitsis, and K. Wilkinson. Data Integration Flows for Business Intelligence. In EDBT, pages 1-11, 2009.

[4] M. Golfarelli, D. Maio, and S. Rizzi. The Dimensional Fact Model: A Conceptual Model for Data Warehouses. IJCIS, pages 215-247, 1998.

[5] M. Golfarelli and S. Rizzi. Data Warehouse Design. Modern Principles and Methodologies. McGraw-Hill, 2009.

[6] B. Hüsemann, J. Lechtenbörger, and G. Vossen. Conceptual Data Warehouse Modeling. In $D M D W$, pages 1-11, 2000.

[7] J. Lechtenbörger and G. Vossen. Multidimensional Normal Forms for Data Warehouse Design. Information Systems, pages 415-434, 2003.
[8] W. Lehner, J. Albrecht, and H. Wedekind. Normal Forms for Multidimensional Databases. In SSDBM, pages 63-72, 1998.

[9] H. Lenz and A. Shoshani. Summarizability in OLAP and Statistical Data Bases. In SSDBM, pages 132-143, 1997.

[10] S. Luján-Mora, P. Vassiliadis, and J. Trujillo. Data Mapping Diagrams for Data Warehouse Design with UML. In $E R$, pages 191-204, 2004.

[11] J. Mazón, J. Lechtenbörger, and J. Trujillo. A Survey on Summarizability Issues in Multidimensional Modeling. DKE, pages 1452-1469, 2009.

[12] J.-N. Mazón and J. Trujillo. An MDA Approach for the Development of Data Warehouses. DSS, pages 41-58, 2008.

[13] L. Muñoz, J.-N. Mazón, and J. Trujillo. Automatic Generation of ETL Processes from Conceptual Models. In DOLAP, pages 33-40, 2009.

[14] O. Romero and A. Abelló. Automating Multidimensional Design from Ontologies. In DOLAP, pages 1-8, 2007.

[15] O. Romero and A. Abelló. Automatic Validation of Requirements to Support Multidimensional Design. DKE, 2010.

[16] O. Romero, D. Calvanese, A. Abelló, and M. Rodriguez-Muro. Discovering Functional Dependencies for Multidimensional Design. In DOLAP, pages 1-8, 2009.

[17] A. Simitsis, K. Wilkinson, U. Dayal, and M. Castellanos. Optimizing ETL Workflows for Fault-Tolerance. In ICDE, pages 385-396, 2010.

[18] D. Skoutas and A. Simitsis. Ontology-Based Conceptual Design of ETL Processes for Both Structured and Semi-Structured Data. IJSWIS, pages 1-24, 2007.

[19] I. Song, R. Khare, and B. Dai. SAMSTAR: A Semi-Automated Lexical Method for Generating STAR Schemas from an ER Diagram. In DOLAP, pages 9-16, 2007.

[20] TPC. TPC-DS specification. Available at: www.tpc.org/tpcds/, 2010

[21] P. Vassiliadis, A. Simitsis, and S. Skiadopoulos. Conceptual modeling for ETL processes. In DOLAP, pages 14-21, 2002.

[22] E. S. K. Yu and J. Mylopoulos. From E-R to "A-R" Modelling Strategic Actor Relationships for Business Process Reengineering. In ER, pages 548-565, 1994. 